\title{
Retinal Detachment Following Nd:YAG Posterior Capsulotomy
}

\author{
L. A. FICKER, ${ }^{1}$ S. VICKERS ${ }^{1}$, M. R. C. CAPON ${ }^{2}$, J. MELLERIO ${ }^{2}$ and R. J. COOLING \\ London
}

\begin{abstract}
Summary
Five hundred and eighty-two patients who underwent Nd:YAG laser posterior capsulotomy at Moorfields Eye Hospital were reviewed retrospectively. Twelve patients ( 2 per cent), nine of whom were previously myopic, subsequently developed rhegmatogenous retinal detachment. Comparison with other studies suggests there is no greater risk of retinal detachment associated with $\mathrm{Nd}$ :YAG laser capsulotomy than with surgical discission. The relevance of the damage mechanisms of Nd:YAG lasers are discussed.
\end{abstract}

With the recent trend towards extracapsular cataract extraction, late posterior capsule opacification with secondary visual impairment is a frequent complication. Wilhelmus and Emery, ${ }^{1}$ reported an incidence of 25 per cent after two years and 50 per cent after five years. Coonan et al. ${ }^{2}$ found 16.7 per cent of patients required capsulotomy over a ten year period with a mean delay of 24.3 months.

Over the past five years the use of an $\mathrm{Nd}$ :YAG laser for secondary posterior capsulotomy has become increasingly popular. Since its introduction, an increasing number of recognised complications have been reported and these have included a transient or sustained rise of intraocular pressure, ${ }^{3,4.5,6}$ damage to the corneal endothelium, ${ }^{7,8}$ pitting of intraocular lenses ${ }^{5,9}$ and retinal detachment. ${ }^{10,11,12,13,14}$

Although the reported incidence of retinal detachment following $\mathrm{Nd}$ :YAG capsulotomy is low (see below), the short interval between capsulotomy and the onset of retinal detachment suggests these two events may be causally linked. ${ }^{10}$ To investigate this possibility, we undertook a retrospective survey of 991 patients who underwent Nd:YAG laser posterior capsulotomy.

\section{Methods and Results}

The case notes of all patients who underwent Nd:YAG laser posterior capsulotomy at Moorfields Eye Hospital between January 1984 and April 1986 were reviewed. A Q-switched Nd:YAG LASAG Sirius Microruptor II laser system with a CGP-1 LASAG contact lens was used in all patients. Of the 991 patients treated, adequate data could be obtained on 582. The minimum follow up in all cases was three months and in 500 patients exceeded six months.

Twelve patients ( 2 per cent) were discovered subsequently to have developed rhegmatogenous retinal detachment. Two thirds of the patients were under the age of 65 years of age and the youngest patient was aged 25 years at the time of capsulo-

Correspondence to: Mr. R. J. Cooling, Surgical Vitreoretinal Unit, Moorfields Eye Hospital, City Road, London EC1V 2PD

${ }^{1}$ Surgical Vitreoretinal Unit, Moorfields Eye Hospital, City Road, London EC1V 2PD

${ }^{2}$ Department of Clinical Ophthalmology, Institute of Ophthalmology, Judd Street, London WC1H 9QA

$\mathrm{Mr}$ Capon is a recipient of a Wellcome Research Grant. 
tomy. Eight patients were found to have low myopia $(<-5 D)$ and one had $-8 D$ of myopia prior to cataract extraction. Nine patients were aphakic and three were pseudophakic. The interval between cataract extraction and capsulotomy ranged from six months to 13 years (mean 22 months), and between capsulotomy and presentation of retinal detachment from five weeks to 18 months, but 9 of the 12 patients developed retinal detachment within six months of capsulotomy.

Traction $\mathrm{U}$ tears at the posterior border of the vitreous base were identified in all but one patient. Five were single tears and seven were multiple tears. In one case a giant retinal tear occurred four months after laser capsulotomy in a 25 year old patient who had undergone limbal lensectomy four years previously.

In addition to the clinical aspects of this study, simultaneous investigations of model eye systems were undertaken to elucidate the extent and relative importance of the putative mechanisms of $\mathrm{Nd}$ :YAG lasers and their relative significance in terms of retinal detachment. A description of the apparatus and techniques has been reported elsewhere ${ }^{15}$ and the relevant findings are discussed here.

\section{Discussion}

In this retrospective study we encountered an incidence of rhegmatogenous retinal detachment of 2 per cent following Nd:YAG laser capsulotomy. Although there was insufficient data on 40 per cent of patients treated in this way, the remaining 582 patients constitute one of the largest series to be reported.

In this study, all but one patient had $U$ tears located at the posterior border of the vitreous base. It is interesting to note that of the 12 affected patients, 9 were myopic suggesting that this is an important contributory factor. This agrees with the experience of Ober et al. ${ }^{12}$ in which 10 of 14 pseudophakic patients who developed retinal detachment following $\mathrm{Nd}$ :YAG capsulotomy were myopic.

Table I Mechanisms of damage by intraocular plasmas

Ionisation

Vapourisation

Cavitation

Mechanical stress waves

Impelled particles

Electromagnetic field stress

Light emission
The patient with a giant retinal tear is, to our knowledge, the only patient reported to have this complication following Nd:YAG laser capsulotomy. This patient had previously had lensectomy and anterior vitrectomy and the giant tear is likely to be related to this event. McLeod ${ }^{16}$ has described three similar cases of giant tears after closed microsurgery and considered the mechanism to be a combination of basal gel incarceration and dynamic vitreoretinal traction.

The occurrence of retinal detachment following Nd:YAG laser capsulotomy subsequent to extracapsular cataract extraction may be attributable to the aphakic state, the effect of the capsulotomy, to the specific damage of the Nd:YAG laser or to an underlying predisposition e.g. myopia.

Some authors ${ }^{10}$ have suggested that disruption of the anterior vitreous face may accelerate gel syneresis with consequent posterior vitreous detachment and subsequent retinal break formation. Support for this concept comes from Lerman et al.,${ }^{17}$ who, using NMR, found marked molecular alterations in the vitreous of monkeys and of rabbits after Nd:YAG irradiation. This was not substantiated by Krauss et al. ${ }^{18}$ using similar investigative techniques. Osterlin ${ }^{19}$ has attributed the vitreous changes following intracapsular surgery or posterior capsulotomy, to reduced levels of vitreous hyaluronic acid compared to phakic eyes with consequent vitreous syneresis.

The possible mechanisms by which plasmas may produce damage are listed in Table I. It is unlikely however that any single mechanism alone will induce vitreous changes sufficient to initiate the development of retinal breaks.

\section{(a) Ionisation}

This is the formation of a plasma and involves stripping electrons from atoms and disrupting molecular bonds. This energetic process gives rise to the subsequent mechanisms listed in the table.

\section{(b) Vapourisation}

Once formed the plasma then produces a very high temperature with local vapourisation of the target tissue. The resultant cloud of expanding gaseous products creates a cavity in 
liquids and solids. These temperature changes do not extend further than a few hundred microns beyond the size of the cavity. ${ }^{21}$

\section{(c) Cavitation}

The eventual collapse of the cavity $100 \mu \mathrm{sec}$ or so later gives up a significant amount of energy mostly in the form of a mechanical stress wave. The presence of interfaces, such as the posterior capsule, complicates the process of collapse-see for example Lauterborn ${ }^{22}$-but any damaging processes will be limited to the anterior vitreous.

\section{(d) Mechanical stress waves}

There are two episodes of stress waves with every Nd:YAG exposure: at the onset and formation of the plasma cavity and at the collapse of the cavity. These stress waves propagate in all directions initially at supersonic velocity but rapidly slow to acoustic velocity within a hundred microns or so. Although the pressure transients may be large, they are rapidly attenuated. Their major action is compression or rarefaction but large molecules and collagen fibrils will experience some shear forces. Any harmful effects would be expected only near the plasma site.

\section{(e) Impelled particles}

Energy released by the collapse of the cavity is transformed into kinetic energy that centrifugally impels debris and bubbles to become potentially harmful missiles. ${ }^{7}$ In the viscid medium of the vitreous these missiles will be halted within a millimetre or so.

\section{(f) Electromagnetic field stress}

This is important only within the focal volume. $^{21}$

\section{(g) Light emission}

A plasma formed within the anterior vitreous will produce negligible irradiance at the retinal surface.

It is unlikely that such confined changes at, or within, the vicinity of the posterior capsule could influence significant volumes of vitreous or exert remote effects on the retina. At just suprathreshold energy inputs, multiple plasmas may form in a string, ${ }^{15}$ but again the extent represents only a small fraction of the vitreous dimensions and does not vitiate any of the above comments.

Though our personal experience is that there is optical breakdown in vitreous gel with Nd:YAG posterior capsulotomy, our figure of 2 per cent compares favourably with that of 6.1 per cent after secondary surgical discission $^{20}$ and suggests that $\mathrm{Nd}$ :YAG laser damage itself does not precipitate retinal detachment.

In summary, most patients who undergo Nd:YAG capsulotomy do not appear to be at an increased risk of retinal detachment compared with those treated by surgical discission. However, there may be a small group of patients, mostly myopes, who are potentially at risk of retinal detachment, not from processes unique to the $\mathrm{Nd}$ :YAG, but from capsulotomy induced by any means.

\section{References}

1 Wilhelmus KR and Emery JM: Posterior capsule opacification following phacoemulsification. Ophthal. Surg. 1980, 11: 264-67.

2 Coonan P, Fung WE, Webster RG, Allen AW and Abbott RL: Incidence of retinal detachment following extracapsular cataract extraction. Ophthalmol. 1985, 192: 1096-101.

${ }^{3}$ Channell MM and Beckman $\mathrm{H}$ : Intraocular pressure changes after Nd:YAG laser posterior capsulotomy. Arch. Ophthalmol. 1984, 102: 1024-6.

${ }^{4}$ Richter CU, Arzeno G, Pappas HR, Steinert RF, Puliafito CA and Epstein DL: Intraocular pressure elevation following Nd:YAG laser posterior capsulotomy. Ophthalmol. 1985, 92: 636-40.

${ }^{5}$ Terry AC, Stark WJ, Maumenee AE and Fagadau W: Nd:YAG laser for posterior capsulotomy. Am. J. Ophthalmol. 1983, 96: 716-20.

${ }^{6}$ Vine AK: Ocular hypertension following Nd:YAG laser capsulotomy: a potentially blinding complication. Ophthal. Surg. 1984, 15: 283-4.

${ }^{7}$ Kerr-Muir MG and Sherrard ES: Damage to the corneal endothelium during Nd:YAG photodisruption. Br' J. Ophthalmol. 1985, 69: 77-85.

${ }^{8}$ Richardson TM, Brown SV, Thomas JV and Simmons RJ: Shock wave effect on anterior segment structures following experimental Nd:YAG laser iridectomy. Ophthalmol. 1985, 92: 1387-95.

${ }^{9}$ Nirankari VS and Richards RD: Complications associated with the use of the Nd:YAG laser. Ophthalmol. 1985, 92: 1371-5.

${ }^{10}$ Fastenberg DM, Schwartz P and Lim HZ: Retinal detachment following Nd:YAG laser capsulotomy. Am. J. Ophthalmol. 1984, 97: 228-91.

${ }^{11}$ Keates RH, Steinert RF, Puliafito CA and Maxwell SK: Long-term follow up of Nd:YAG laser pos- 
terior capsulotomy. Am. Intra-ocular Implant Soc. J. 1984, 10: 164-8.

12 Ober RR, Wilkinson CP, Fiorie JB and Maggiano JM: Rhegmatogenous retinal detachments after Nd:YAG laser capsulotomy in phakic and pseudophakic eyes. Am. J. Ophthalmol. 1986, 101: 81-9.

${ }^{13}$ Stark WJ, Worthen D, Holladay JT and Murray G: Neodymium:YAG lasers: an FDA report. Ophthalmol. 1985, 92: 209-12.

${ }^{14}$ Winslow RL and Taylor BC: Retinal complications following YAG capsulotomy. Ophthalmol. 1985, 92: 785-9.

${ }^{15}$ Capon MRC and Mellerio J: Nd:YAG lasers: plasma characteristics and damage mechanisms. Lasers in Ophthalmol. 1986, (in press).

${ }^{16}$ McLeod D: Giant retinal tears after central vitrectomy. Br. J. Ophthmol. 1985, 69: 96-8.

${ }^{17}$ Lerman S, Thrasher B and Moran M: Vitreous changes after Nd:YAG laser irradiation of the posterior lens capsule or mid-vitreous. Am. J. Ophthalmol. 1984, 97: 470-5.

${ }^{18}$ Krauss JM, Puliafito CA, Miglior S, Steinert RF and Cheng H-M: Vitreous changes after Nd:YAG laser photodisruption. Arch. Ophthalmol. 1986, 104: 592-7.

${ }^{19}$ Osterlin S: On the molecular biology of the vitreous in the aphakic eye. Acta Ophthalmologica 1977, 55: 353-61.

${ }^{20}$ Fung WE, Coonan P and Ho BT: Incidence of retinal detachment following extracapsular cataract extraction. Retina 1981, 1: 232-7.

${ }^{21}$ Mainster MA, Sliney DH, Belcher CD and Buzney SM: Laser photodisruptors: damage mechanisms, instrument design and safety. Ophthalmol. 1983, 90: 973-91.

${ }^{22}$ Lauterborn W: Cavitation and coherent optics. In Lauterborn W Ed. Cavitation and inhomogeneities in underwater acoustics. Berlin: Springer-Verlag 1980, pp. 3-12. 